\title{
Network Pharmacology-Based Approach to Investigate the Mechanisms of Piwei-Peiyuan Decoction in the Treatment of Gastric Carcinoma
}

\section{Bei Pei}

Anhui University of Traditional Chinese Medicine

\section{Zi'ang Wen}

Anhui Medical University

Yanmin Zheng

Hefei Institutes of Physical Science

Xuejun Li ( $\square$ lixuejun@ahtcm.edu.cn )

Second Affiliated Hospital of Anhui Medical University

Original Article

Keywords: Piwei-Peiyuan Decoction, Gastric Cancer, Molecular Docking, Traditional Chinese Medicine, Treatment

Posted Date: February 10th, 2021

DOI: https://doi.org/10.21203/rs.3.rs-208530/v1

License: (c) (i) This work is licensed under a Creative Commons Attribution 4.0 International License. Read Full License 


\section{Abstract \\ Purpose}

Piwei-Peiyuan Decoction is a traditional Chinese medicine decoction, which has shown promising treatment in gastric carcinoma. However, the molecular mechanisms of gastric carcinoma (GC) have not been systematically revealed yet. In this work, a network pharmacology research was conducted to analyze mechanisms of Piwei-Peiyuan Decoction, aiming to provide a basis for the development and application of new drugs in treatment of GC.

\section{Methods}

In this study, a network pharmacology approach was used to predict targets, construct network maps and analyze relevant signaling pathways. We discovered active chemical ingredients and their targets in the Piwei-Peiyuan Decoction by the TCMSP and ADME database. Then we found main targets of GC by GeneCards (https://www.genecards.org), OMIM (http://www.omim.org) and DRUGBANK (https://go.drugbank.com) databases. According to the information of ingredients and GC, a PPI network was performed by the String (https://string-db.org) database. And a targets-pathways interaction network was constructed by Cytoscape (version 3.7.2), a bioinformatics software used for data visualization and integration.

\section{Results}

Our result indicated that the main active ingredients of Piwei-Peiyuan Decoction in treating GC are Isorhamnetin, Kaempferol, Quercetin and Luteolin, and main active targets are CASP-3, MAPK1, MYC, AKT1, P53. We found the mechanism of Piwei-Peiyuan Decoction for treating GC is mainly to regulate PI3K-Akt signaling pathway, MAPK signaling pathway, Ras signaling pathway, IL-17 signaling pathway, HIF-1a signaling pathway to inhibit cell proliferation, promote apoptosis and then exert anti-cancer effects.

\section{Conclusion}

This study preliminarily analyzed the multi-component, multi-target and multi-path mechanism of PeiweiPeiyuan Decoction in the treatment of gastric carcinoma, providing a theoretical basis for its clinical application.

\section{Background}

As a type of malignancy that origin from epithelial cells of gastric mucosa. Almost $95 \%$ of GC is adenocarcinoma. GC is a malignancy with the highest morbidity and mortality in China after lung cancer, 
breast cancer and colon cancer, its 5 -year survival rate is only $29 \%$. Although the morbidity of GC in China has declined in recent years, the mortality has not decreased significantly ${ }^{12}$. The morbidity of GC still ranks second and fifth among all malignancy among men and women, and the mortality ranks third and second in China $^{34}$. At present, surgery is main treatment of GC. Perioperative chemotherapy and radiotherapy are main treatments to improve the surgical resection rate ${ }^{56}$. However, since the high recurrence rate after surgery and the complications of other treatments, the final prognosis of patients is poor $^{7}$.

Traditional Chinese medicine has been widely used in clinical treatment of cancer in Asian countries, especially China and Japan $^{9}$. As many clinical trials on comprehensive cancer treatment continue to be conducted, we have found that TCM not only relieves the discomfort caused by surgery and other treatments, but also alleviates the clinical symptoms of patients and improves their immune function and postoperative survival rate ${ }^{10-12}$. The results of a Meta-analysis on TCM treatment improves the survival rate of GC patients in Taiwan ${ }^{13}$ showed that the median survival time of patients who received TCM treatment was longer than that of patients who did not receive TCM treatment, suggesting that TCM treatment improves the medium overall survival rate of GC patients. TCM has its unique advantages in treating cancer, which can effectively inhibit tumor spread and metastasis, reduce toxicity of chemotherapy drugs and enhance immunity. For thousands of years, TCM has been widely used to treat various diseases because of its multi-target, low price and few side effects ${ }^{14}$. TCM also helps to improve the quality of life among GC patients ${ }^{15}$. Piwei-Peiyuan Decoction is a prescription developed by Professor Xuejun Li on the basis of Dongheng Li's theory of spleen and stomach diseases, combined with the clinical experience of Professor Ma Jun, a national famous TCM doctor. This prescription consists of six herbs including Bai Zhu, Huang Qi, Xiang Fu, Gui Zhi, Bai Shao and Liu Jinu. In our hospital, PiweiPeiyuan Decoction has shown outstanding efficacy in treatment of GC, which can effectively reduce the clinical symptoms of GC patients. However, its pharmacological mechanisms and compounds are still unclear. It is necessary for us to discover the compounds and key targets of Piwei-Peiyuan Decoction in treatment of GC.

Based on genomic, proteomic and pharmacological information $₫ a$ "disease-gene" biological network and a "drugs-targets" biological network can be constructed through a network pharmacology approach. After analysis, we can understand the mechanisms of drugs in treatment of diseases systematically and comprehensively ${ }^{16} 17$. Through the network pharmacology method, the main targets of Piwei-Peiyuan Decoction can be identified, which is helpful to further investigate the mechanisms of Piwei-Peiyuan Decoction in treating GC and provide an important theoretical basis for its clinical treatment.

\section{Methods}

2.1 Predicting Targets of Piwei-Peiyuan Decoction 
To obtain the targets of Piwei-Peiyuan Decoction, TCMSP database (https://tcmspw.com/tcmsp.php) were used. TCMSP ${ }^{18}$ contains 499 Chinese herbal medicines registered in the Chinese Pharmacopoeia, including information of 29,384 ingredients, 3,311 targets and 837 related diseases. TCM are mostly oral medication, according to oral bioavailability (OB) and drug-likeness point (DL), active ingredients and related targets can be selected ${ }^{19} 20$. We took $\mathrm{OB} \geq 30 \%$ and $\mathrm{DL} \geq 0.18$ as a standard, discovered the active ingredients and related targets. Then the names of those identified targets were sent to UNIPROT database (http://www.uniprot.org) for normalization.

\subsection{Collecting Targets Related to Gastric Carcinoma}

To obtain the disease-related gene, Genecards database (https://www.genecards.org) and OMIM database (http://www.omim.org) were used. GeneCards ${ }^{21}$ is a comprehensive and authoritative database of human gene information that has been widely used for nearly 26 years. Its information is automatically discovered and integrated from more than 80 digital sources, including more than 73,000 human genes. We set "Gastric Carcinoma" as keywords, selected targets with hit scores greater than median (1.55) and deleted the repeated targets. Finally, GC-related targets were obtained.

\subsection{Protein-Protein-Interaction Analysis}

Protein-Protein-Interaction (PPI) is fundamental for most biological processes in a living cell and is crucial for understanding cell physiology in normal and disease states. Our PPI network mapping was performed on obtained bioactive ingredients and disease targets using STRING database (http://string$\mathrm{db}$. org) with the species limited to "homo sapiens". A targets-pathways interaction network were constructed by Cytoscape (version 3.7.2), a bioinformatics software used for data visualization and integration 2223 . Cytoscape is the most powerful tool when large amounts of information about the interrelationship of DNA, protein and signal pathways need to be analyzed.

\subsection{GO and KEGG Pathway Enrichment}

The Gene Ontology (GO) provides information for functional genomics and defines the concepts relating to gene functions ${ }^{24}$. The Kyoto Encyclopedia of Genes and Genomes (KEGG) is a database that is famous for its pathway information ${ }^{25}$. It is a powerful database for systematic analysis of gene function, which links genomic information to biological function information. In order to investigate the biological effects of Peiwei-Peiyuan Decoction, GO analysis and KEGG pathway enrichment analysis were conducted and calculated by STRING database (http://string-db.org). The enriched GO terms and pathways having a corrected $P$ value of less than 0.01 were selected for further analysis.

\subsection{Molecular Docking}

Based on the information from network maps of Piwei-Peiyuan Decoction and GC, we selected main ingredients and targets of Piwei-Peiyuan Decoction for molecular docking experiment. The threedimensional (3D) structure diagrams of these compounds of Piwei-Peiyuan Decoction were downloaded 
through the TCMSP database and imported into the AutodockTools1.5.6 software for hydrogenation and energy optimization, then the mol2 format files were saved. We add the charge and display rotatable keys of these compounds and the files were saved in pdbqt format. Next, the protein crystal structures corresponding to the target genes were downloaded from the PDB database, imported into PyMOL software to remove water molecules and heteromolecules, imported into AutoDockTools-1.5.6 software to add hydrogen atoms, saved to pdbqt format. Finally, the compound is used as a ligand, and the protein corresponding to the target gene is used as a receptor for molecular docking. Autodock vina 1.1.2 and PyMol software were used to analyze and interpret the results.

\section{Results}

3.1 Ingredients and Targets Analysis

In our study, the TCMSP database was used to predicted the ingredients of Piwei-Peiyuan Decoction initially. We obtained 55 ingredients for Bai Zhu, 87 ingredients for Huang Qi, 104 ingredients for Xiang Fu, 220 ingredients for Gui Zhi, 85 ingredients for Bai Shao and 46 ingredients for Liu Jinu. Then we deleted the active ingredients without matched targets and selected the active ingredients from 6 herbs according to oral bioavailability $(\mathrm{OB})$ and drug likeness $(\mathrm{DL})$ values $(\mathrm{OB} \geq 30 \%$ and $\mathrm{DL} \geq 0.18)$. Eventually, 67 active ingredients such as quercetin, isorhamnetin, luteolin and kaempferol were discovered (Table.1), and they all have hepatoprotective, anti-inflammatory, antibacterial, analgesic and antitumor effects ${ }^{26-29}$.

\section{Table 1. Active ingredients of Piwei-Peiyuan Decoction}




\begin{tabular}{|c|c|c|c|c|}
\hline Medicine & MOL ID & MOL NAME & $\mathrm{OB}(\%)$ & DL \\
\hline Bai Zhu & MOL000033 & $\begin{array}{l}\text { (3S,8S,9S,10R,13R,14S,17R)-10,13-dimethyl-17- } \\
{[(2 R, 5 S)-5-p r o p a n-2-y l o c t a n-2-} \\
\text { yl]-2,3,4,7,8,9,11,12,14,15,16,17-dodecahydro-1H- } \\
\text { cyclopenta[a]phenanthren-3-ol }\end{array}$ & 36.23 & 0.78 \\
\hline Bai Zhu & MOL000020 & 12-senecioyl-2E,8E,10E-atractylentriol & 62.4 & 0.22 \\
\hline Bai Zhu & MOL000021 & 14-acetyl-12-senecioyl-2E,8E,10E-atractylentriol & 60.31 & 0.31 \\
\hline Bai Zhu & MOL000022 & 14-acetyl-12-senecioyl-2E,8Z,10E-atractylentriol & 63.37 & 0.30 \\
\hline Bai Zhu & MOL000049 & $3 \beta$-acetoxyatractylone & 54.07 & 0.22 \\
\hline Bai Zhu & MOL000072 & $8 \beta$-ethoxy atractylenolide $\mathbb{Z}$ & 35.95 & 0.21 \\
\hline Bai Zhu & MOL000028 & a-Amyrin & 39.51 & 0.76 \\
\hline \multirow{2}{*}{$\begin{array}{l}\text { Huang } \\
\text { Qi }\end{array}$} & MOL000438 & (3R)-3-(2-hydroxy-3,4-dimethoxyphenyl)chroman-7-ol & 67.67 & 0.26 \\
\hline & MOL000033 & $\begin{array}{l}\text { (3S,8S,9S,10R,13R,14S,17R)-10,13-dimethyl-17- } \\
{[(2 R, 5 S)-5-p r o p a n-2-y l o c t a n-2-} \\
\text { yl]-2,3,4,7,8,9,11,12,14,15,16,17-dodecahydro-1H- } \\
\text { cyclopenta[a]phenanthren-3-ol }\end{array}$ & 36.23 & 0.78 \\
\hline \multirow{5}{*}{$\begin{array}{l}\text { Huang } \\
\text { Qi }\end{array}$} & MOL000380 & $\begin{array}{l}(6 a R, 11 a R)-9,10-d i m e t h o x y-6 a, 11 \text { a-dihydro-6H- } \\
\text { benzofurano[3,2-c]chromen-3-ol }\end{array}$ & 64.26 & 0.42 \\
\hline & MOL000442 & 1,7-Dihydroxy-3,9-dimethoxy pterocarpene & 39.05 & 0.48 \\
\hline & MOL000371 & 3,9-di-O-methylnissolin & 53.74 & 0.48 \\
\hline & MOL000374 & 5'-hydroxyiso-muronulatol-2',5'-di-O-glucoside & 41.72 & 0.69 \\
\hline & MOL000378 & 7-0-methylisomucronulatol & 74.69 & 0.30 \\
\hline \multirow{3}{*}{$\begin{array}{l}\text { Huang } \\
\mathrm{Qi}\end{array}$} & MOL000379 & 9,10-dimethoxypterocarpan-3-O- $\beta$-D-glucoside & 36.74 & 0.92 \\
\hline & MOL000387 & Bifendate & 31.10 & 0.67 \\
\hline & MOL000417 & Calycosin & 47.75 & 0.24 \\
\hline \multirow{3}{*}{$\begin{array}{l}\text { Huang } \\
\text { Qi }\end{array}$} & MOL000433 & FA & 68.96 & 0.71 \\
\hline & MOL000392 & Formononetin & 69.67 & 0.21 \\
\hline & MOL000296 & Hederagenin & 36.91 & 0.75 \\
\hline $\begin{array}{l}\text { Huang } \\
\mathrm{Qi}\end{array}$ & MOL000398 & Isoflavanone & 109.99 & 0.30 \\
\hline \multirow{3}{*}{$\begin{array}{l}\text { Huang } \\
\text { Qi }\end{array}$} & MOL000439 & Isomucronulatol-7,2'-di-O-glucosiole & 49.28 & 0.62 \\
\hline & MOL000354 & Isorhamnetin & 49.60 & 0.31 \\
\hline & MOL000239 & Jaranol & 50.83 & 0.29 \\
\hline $\begin{array}{l}\text { Huang } \\
\text { Qi }\end{array}$ & MOL000422 & Kaempferol & 41.88 & 0.24 \\
\hline
\end{tabular}


Huang

Qi

Huang

Qi

Huang

Qi

Huang

Qi

Huang

Qi

Huang

Qi

Huang

Qi

Huang

Qi

Huang

Qi

Huang

Qi

Huang

Qi

Huang

Qi

\begin{tabular}{|cllrl|}
\hline Bai Shao & MOL001930 & Benzoyl paeoniflorin & 31.27 & 0.75 \\
\cline { 2 - 5 } Bai Shao & MOL000359 & Sitosterol & 36.91 & 0.75 \\
\cline { 2 - 5 } Bai Shao & MOL000358 & Beta-sitosterol & 36.91 & 0.75 \\
Bai Shao & MOL000422 & Kaempferol & 41.88 & 0.24 \\
Bai Shao & MOL001919 & $\begin{array}{l}\text { (3S,5R,8R,9R,10S,14S)-3,17-dihydroxy-4,4,8,10,14- } \\
\text { pentamethyl-2,3,5,6,7,9-hexahydro-1H- } \\
\text { cyclopenta[a]phenanthrene-15,16-dione }\end{array}$ & 43.56 & 0.53 \\
& MOL001921 & Lactiflorin & 49.12 & 0.80 \\
\cline { 2 - 5 } & MOL001924 & Paeoniflorin & 53.87 & 0.79 \\
\cline { 2 - 5 } & MOL000492 & (+)-Catechin & 54.83 & 0.24
\end{tabular}




\begin{tabular}{|c|c|c|c|c|}
\hline \multirow{2}{*}{$\begin{array}{l}\text { Bai Shao } \\
\text { Bai Shao } \\
\text { Bai Shao }\end{array}$} & MOL000211 & Mairin & 55.38 & 0.78 \\
\hline & MOL001910 & $\begin{array}{l}\text { 11alpha,12alpha-epoxy-3beta-23-dihydroxy-30- } \\
\text { norolean-20-en-28,12beta-olide }\end{array}$ & 64.77 & 0.38 \\
\hline \multirow{3}{*}{$\begin{array}{l}\text { Bai Shao } \\
\text { Bai Shao }\end{array}$} & MOL001928 & albiflorin_qt & 66.64 & 0.33 \\
\hline & MOL001925 & Paeoniflorin_qt & 68.18 & 0.40 \\
\hline & MOL001918 & Paeoniflorgenone & 87.59 & 0.37 \\
\hline \multicolumn{5}{|l|}{ Bai Shao } \\
\hline \multicolumn{5}{|l|}{ Bai Shao } \\
\hline \multicolumn{5}{|l|}{ Bai Shao } \\
\hline Xiang Fu & MOL003044 & Chryseriol & 35.85 & 0.27 \\
\hline Xiang Fu & MOL000354 & Isorhamnetin & 49.60 & 0.31 \\
\hline Xiang Fu & MOL003542 & 8-Isopentenyl-kaempferol & 38.04 & 0.39 \\
\hline Xiang Fu & MOL000358 & Beta-sitosterol & 36.91 & 0.75 \\
\hline Xiang Fu & MOL000359 & Sitosterol & 36.91 & 0.75 \\
\hline \multirow[t]{2}{*}{ Xiang Fu } & MOL004027 & 1,4-Epoxy-16-hydroxyheneicos-1,3,12,14,18-Pentaene & 45.10 & 0.24 \\
\hline & MOL004053 & Isodalbergin & 35.45 & 0.20 \\
\hline \multirow{2}{*}{$\begin{array}{l}\text { Xiang Fu } \\
\text { Xiang Fu }\end{array}$} & MOL004058 & Khell & 33.19 & 0.19 \\
\hline & MOL004059 & Khellol glucoside & 74.96 & 0.72 \\
\hline \multirow{2}{*}{$\begin{array}{l}\text { Xiang Fu } \\
\text { Xiang Fu }\end{array}$} & MOL010489 & Resivit & 30.84 & 0.27 \\
\hline & MOL004068 & Rosenonolactone & 79.84 & 0.37 \\
\hline Xiang Fu & MOL004071 & Hyndarin & 73.94 & 0.64 \\
\hline $\begin{array}{l}\text { Xiang Fu } \\
\text { Xiang Fu }\end{array}$ & MOL004074 & Stigmasterol glucoside_qt & 43.83 & 0.76 \\
\hline Xiang Fu & MOL004077 & Sugeonyl acetate & 45.08 & 0.20 \\
\hline Xiang Fu & MOL000422 & Kaempferol & 41.88 & 0.24 \\
\hline Xiang Fu & MOL000449 & Stigmasterol & 43.83 & 0.76 \\
\hline Xiang Fu & MOL000006 & Luteolin & 36.16 & 0.25 \\
\hline Xiang Fu & MOL000098 & Quercetin & 46.43 & 0.28 \\
\hline Gui Zhi & MOL000073 & ent-Epicatechin & 48.96 & 0.24 \\
\hline
\end{tabular}




\begin{tabular}{|cllll|} 
Gui Zhi & MOL000492 & (+)-Catechin & 54.83 & 0.24 \\
\cline { 2 - 5 } Gui Zhi & MOL001736 & (-)-Taxifolin & 60.51 & 0.27 \\
\cline { 2 - 5 } Gui Zhi & MOL004576 & Taxifolin & 57.84 & 0.27 \\
Gui Zhi & MOL000359 & Sitosterol & 36.91 & 0.75 \\
Gui Zhi & MOL011169 & Peroxyergosterol & 44.39 & 0.82 \\
\hline Liu Ji'nu & MOL001733 & EUPATORIN & 30.23 & 0.37 \\
Liu Ji'nu & MOL000358 & Beta-sitosterol & 36.91 & 0.75 \\
Liu Ji'nu & MOL000006 & Luteolin & 36.16 & 0.25 \\
Liu Ji'nu & MOL008127 & Ermanin & 58.95 & 0.30 \\
Liu Ji'nu & MOL008135 & 3,4-di-O-caffeoylquinic acid & 49.62 & 0.69 \\
\cline { 2 - 5 }
\end{tabular}

\subsection{Targets of Gastric Carcinoma and Piwei-Peiyuan Decoction}

9877 targets of GC were obtained by Genecards database. We selected targets with hit scores greater than median 1.55. Finally, after integrating the target information in the database, 4938 targets were saved.

\subsection{Construction of Interaction Network Maps}

Based on the targets of Piwei-Peiyuan Decoction and GC, 181 common targets shown in the venn diagram were recognized as targets of Piwei-Peiyuan Decoction in treatment of GC (Figure 1). A PPI network was constructed based on STRING database (Figure 2), and a Targets-Pathways Interaction Network was built based on Cytoscape 3.7.1 (Figure 3). The obtained targets, such as MAPK1, TP53, IL-1, Fox and Bax, are all involved in anti-inflammatory, cell proliferation-promoting, angiogenic, and anti-tumor processes $^{30-33}$.

\subsection{GO and KEGG Pathway Enrichment Analysis}

GO enrichment analysis consists three parts: Biological Process, Molecular Function and Cellular Component. We sent 181 targets information to the STRING database for GO analysis. Finally, 2311 biological process terms, 139 cellular component terms and 259 molecular function terms were found (Figure 4). Based on the correct $P$ value, we found the top 5 terms in cellular component were cell (174), intracellular (166), organelle (151), intracellular organelle (150), membrane-bounded organelle (149). And top 5 terms in biological processes were cellular process (178), biological regulation (174), response to stimulus (173), regulation of biological process (168), regulation of cellular process (165). Top 5 terms in molecular funtions were binding (173), protein binding (145), ion binding (111), catalytic activity (96), organic cyclic compound binding (92). 
To further uncover the potential pharmacological mechanisms of Piwei-Peiyuan Decoction against GC, pathway analysis was conducted to explore the potential pathways affected by Peiwei-Peiyuan Decoction. 190 pathways were found by KEGG database, we selected top 20 pathways and draw a bubble diagram as follows (Figure 5). Combining the pathogenesis of GC, the pathways which have no association with GC were removed. Finally, 6 remarkable terms were found to be the related pathways in treatment of GC. Results demonstrated that "Pathway in cancer", "PI3K-Akt signaling pathway", "MAPK signaling pathway", "Ras signaling pathway", "IL-17 signaling pathway", "HIF-1alpha signaling pathway" and "TNF signal pathway" were obviously enriched. According to the KEGG analysis, We found "Pathway in cancer" is the signal pathway that contains the most targets (72 targets), the network of "Pathway in cancer" is shown below (Figure 6). These signaling pathways are closely related to cell differentiation, proliferation, apoptosis and angiogenesis, most of which play a key role in the development and progression of cancer. The molecular functions and biological processes were closely related to the occurrence and development of GC, which indicated that Piwei-Peiyuan Decoction can treat GC through multiple targets and pathways. Our research shows that Piwei-Peiyuan Decoction acts as a treatment for GC mainly through the coordinated regulation of cancer-related signal pathways. For example, PI3K-Akt signaling pathway is a key pathway that regulates cell proliferation, differentiation and metastasis during the development of cancer ${ }^{34}$, and activated AKT can lead to apoptosis by participating in the regulation of cellular protein expression, then ultimately inhibiting cell proliferation ${ }^{35}$. It has been shown that the abnormal expression of P13K-Akt signaling pathway in GC tissues is closely related to the development and prognosis of tumor, P13K-AKT signal pathway plays an important role in regulating the proliferation, invasion and metastasis of tumor cells. Meanwhile, the high expression of P13K-Akt signaling pathway in GC is related to the degree of tumor differentiation, its expression level was positively correlated with the malignancy of $\mathrm{GC}^{36-38}$. MAPK signal pathway is expressed in almost all eukaryotes. It participates in cellular activities such as gene expression, cell proliferation and apoptosis, playing a key role in cellular activities $^{39}$. ERK signaling pathway is a significant part of MAPK signal pathway, the gene mutations or abnormal activation in ERK signal pathway can lead to development of cancer ${ }^{40}$. Ras is a binding kinase in the upstream of ERK/MAPK signal pathway, its point mutations can lead to dysregulation of ERK/MAPK signal pathway and abnormal cellular activity, which in turn lead to migration and invasion of cancer cells and ultimately induces the development of tumor ${ }^{4142}$. IL-17 is an inflammatory factor with great anti-inflammatory effects. It participates in the processes of cell proliferation and differentiation, immune regulation, and tumor growth. A study ${ }^{43}$ showed that the median serum IL-17 level in GC patients was significantly higher than that in controls ( $9.04 \mathrm{VS} 8.07 \mathrm{pg} / \mathrm{ml}, \mathrm{p}=0.01)$, which shows that serum IL-17 level can be used as a new potential indicator for GC diagnosis. Xu J et al ${ }^{44}$ found that IL-17-mediated downregulation of LCN2 expression inhibited the proliferation, migration, and invasion of GC cells by regulating SLPI. In addition to IL-17A, a member of the IL-17 family, several experiments have confirmed that IL-17B/IL-17 receptor B (IL-17RB) can inhibit growth and progression of tumor. A recent study ${ }^{45}$ by Bastid $\mathrm{J}$ et al showed that IL-17B promotes tumor progression by promoting the secretion of chemokines and cytokines and thereby dramatically altering the tumor microenvironment. Furthermore, $\mathrm{n}$ GC patients, higher serum IL-17B levels have been shown to be strongly associated with poor prognostic outcomes. 
Therefore, it is clear that IL-17 levels are closely associated with the development of GC. However, few pharmacological findings have been reported for the treatment of GC with Piwei-Peiyuan Decoction. Therefore, more experiments should be conducted to validate our findings in the future.

\subsection{Results of Molecular Docking Experiment}

Molecular docking was used for verification of interaction between ingredient and its target gene. The results of molecular docking scores of the ingredients and target genes we selected in this study were shown in Table.2, and the optimal schematic of molecular docking was shown in Figure.7.

From the results, the lowest binding free energy of caspase- 3 and kaempferol is $-6.1 \mathrm{kcal} / \mathrm{mol}$. There is one hydrogen bond between amino acid THR62 and kaempferol. The lowest binding free energy of caspase- 3 and luteolin is $-6.1 \mathrm{kcal} / \mathrm{mol}$. There are hydrophobic interactions between amino acid THR62, SER251 and luteolin. In addition, the lowest binding free energy of MAPK1 and luteolin is $-7.9 \mathrm{kcal} / \mathrm{mol}$. There are hydrophobic interactions between amino acid LYS54, GLN105, MET108, ASP167 and luteolin. The lowest binding free energy of caspase -3 and quercetin is $-6.0 \mathrm{kcal} / \mathrm{mol}$. There are two hydrophobic bond forces between amino acid THR62 and quercetin. Moreover, The lowest binding free energy of MAPK1 and quercetin is $-8.1 \mathrm{kcal} / \mathrm{mol}$. There are two hydrophobic bond forces between amino acid ASP106, MET108 and quercetin. All compounds showed a compact binding pattern to the protein active pocket, and these interactions enabled the proteins to form stable complexes with all compounds. In this study, molecular docking experiment gives explanation for the way of protein-compound interactions and lays the theoretical foundation for further studies of Piwei-Periyuan Decoction in treatment of GC.

\section{Table.2 Scores of Molecular Docking}

\begin{tabular}{|lll|}
\hline Receptor_Name & Ligand_Name & Scores $(\mathrm{kcal} / \mathrm{mol})$ \\
\hline CASP3 & kaempferol.pdbqt & -6.1 \\
\hline CASP3 & luteolin.pdbqt & -6.1 \\
\hline CASP3 & quercetin.pdbqt & -6.0 \\
\hline MAPK1 & luteolin.pdbqt & -7.9 \\
\hline MAPK1 & quercetin.pdbqt & -8.1 \\
\hline
\end{tabular}

Note: A higher absolute value of scores means that the binding of receptor protein and active ingredient is more stable.

\section{Conclusion}


In this study, we investigated the mechanism of Piwei-Peiyuan Decoction in treatment of GC using a network pharmacology approach. The results showed that Piwei-Peiyuan Decoction exerted its excellent pharmacological effects in treatment of GC through various pathways, such as regulation of cell cycle, promotion of tumor cell apoptosis and immunomodulation. Our study also provided a theoretical basis for the clinical application of Piwei-Peiyuan Decoction, which is of clinical value to further explore the role of TCM in prevention and treatment of cancer in the future.

\section{Discussion}

$\mathrm{GC}$ is one of the most common cancers in the world. At present, the mechanism of GC is not fully understood. However, according to the patient's pathogenesis and clinical symptoms, in the theory of TCM, GC is caused by weakness of body, bad dietary habits and emotional disorders. Several studies have shown that surgery, radiotherapy and chemotherapy for GC are ineffective, while TCM treatment has its own advantages. It has great potential in improving immunity of body and alleviating symptoms caused by $\mathrm{GC}^{46}$. Although Piwei-Peiyuan Decoction has been widely used in treatment of GC, there is no research on its specific mechanisms in treatment of GC. In our study, a network pharmacology method was used to predict and discover the potential mechanisms of Piwei-Peiyuan Decoction in treatment of GC. From this experiment, it can be seen that quercetin, isorhamnetin, luteolin and kaempferol may be the key components of Piwei-Peiyuan Decoction, and cell cycle regulation-related proteins play an important role in the development of GC. Quercetin is a bioflavonoid with anti-inflammatory, antiviral, and anti-tumor effects, and it has been shown to exert inhibitory activity on growth of GC cells through various mechanisms ${ }^{47-49}$. Quercetin can regulate the expression of caspases family and $\mathrm{Bcl}-2$ family proteins to induce cell apoptosis through MAPK, ERK, PI3K, PKC and other signaling pathways ${ }^{50-53}$. Shang HS et al ${ }^{54}$ found that quercetin could induce apoptosis in AGS cells by causing changes in cell morphology and thus reduce the overall survivability of GC cells. Kaempferol is an important flavonoid widely found in vegetables and fruits, which induces cell cycle arrest and promotes cell apoptosis ${ }^{55-59}$. It has been shown to inhibit the proliferation and metastasis of a variety of tumor cells. Yang $L$ et al ${ }^{60}$ found that kaempferol treats GC mainly through three key targets, ESR1, EGFR and SRC. The expression levels of EGFR and SRC were differentially elevated in GC tissues, and high expression of these targets could directly affect the prognostic survival of GC patients. It was found that apoptosis and G2/M phase cell cycle arrest were observed in tumor cells after kaempferol treatment, and the expression levels of G2/M cell cycle regulators, cyclin $\mathrm{B} 1, \mathrm{Cdk} 1$ and $\mathrm{Cdc} 25 \mathrm{C}$ were significantly reduced. In addition, after kaempferol treatment, Bcl-2 expression levels were significantly reduced while Bax expression levels were increased, these changes led to the upregulation of caspase- 3 and caspase- 9 , which promoted apoptosis and finally inhibited the development of $\mathrm{GC}^{61}$. Isorhamnetin is one of the most important active ingredients in hippophae rhamnoides fruits and ginkgo biloba leaves, with a wide range of pharmacological activity, which can exert anti-inflammatory, anti-tumor and antioxidant effects through the regulation of PI3K/AKT/PKB, NF-KB, MAPK signaling pathways and the expression of related cytokines and kinases ${ }^{62}$. Ramachandran $L$ et al ${ }^{63}$ found that isorhamnetin can reduce the migratory and invasive properties of GC cells by regulating peroxisome proliferator-activated receptor y (PPAR-y), thereby inhibiting GC cell 
proliferation and inducing cell apoptosis. In addition, the combination of isorhamnetin and chemotherapy drugs can enhance the inhibition of tumor cell proliferation. Luteolin is a common flavonoid in plants, which significantly inhibits cell cycle progression, proliferation, migration, invasion and promotes cell apoptosis $^{64}$. Luteolin exerts its anti-tumor, antioxidant, and anti-inflammatory effects mainly through the regulation of signaling pathways such as Notch1, PI3K, AKT and ERK signal pathway. A study has been found that high expression of Notch1 was closely associated with low overall survival rate of GC patients ${ }^{65}$. However, Luteolin could inhibit GC progression by inhibiting Notch1 signaling pathway and reversing epithelial-mesenchymal transition (EMT). It suggests that luteolin may be an effective antitumor compound in treatment of GC. KEGG pathway enrichment analysis showed that the target genes of Piwei-Peiyuan Decoction for the treatment of GC were mainly involved in Pathway in cancer, PI3K-Akt signaling pathway, MAPK signaling pathway, IL-17 signaling pathway, Ras signaling pathway, HIF-1 signaling pathway and TNF signaling pathway. In summary, it can be seen that the mechanism of PiweiPeiyuan Decoction in treatment of GC is to inhibit cell proliferation and promote cell apoptosis by interfering with tumor-related signaling pathways, thus exerting anti-tumor effects.

\section{Abbreviations}

AKT

Protein Kinase B

Bai Shao

Radix Paeoniae Alba

Bai Zhu

Rhizoma Atractylodis Macrocephalae

DL

Drug Likeness

EGFR

Epidermal Growth Factor Receptor

ESR

Estrogen Receptor

GC

Gastric Carcinoma

GO

Gene Ontology

Gui Zhi

Ramulus Cinnamomi

HIF

Hypoxia Inducible Factor

Huang Qi

Radix Astragali

IL 
Interleukin

KEGG

Kyoto Encyclopedia of Genes and Genomes

Liu Jinu

Herba Artemisiae Anomalae

MAPK

Mitogen-activated Protein Kinase

OB

Oral Bioavailability

OMIM

Online Mendelian Inheritance in Man

PKB

Protein Kinase B

PPI

Protein-Protein Interaction Analysis

TCM

Traditional Chinese Medicine

TCMSP

Traditional Chinese Medicine Systems Pharmacology Database and Analysis Platform

TNF

Tumor Necrosis Factor

Xiang Fu

Cyperus Rotundus

\section{Declarations}

\section{Contributions}

$\mathrm{XL}$ conceived and designed research. BP, ZW and $\mathrm{YZ}$ conducted experiments and collected data. BP analyzed data and wrote the manuscript. All authors read and approved the manuscript.

Besides, we declare that all data were generated in-house and that no paper mill was used.

\section{Corresponding Author}

Correspondence to Xuejun Li.

\section{Ethics Approval}

Not applicable.

\section{Conflicts of Interest}


There are no conflicts of interest to declare.

\section{Funding}

This work was supported by the Clinical Efficacy and Safety Observation of Shisanweihezhong pills in the Treatment of Elderly Functional Dyspepsia Project of Anhui, China (grant 1804h08020293); The Fourth Batch of National Research and Training Programs for the Outstanding Traditional Chinese Medicine Talents of China.

\section{References}

1. Bray F, Ferlay J, Soerjomataram I, et al. Global cancer statistics 2018: GLOBOCAN estimates of incidence and mortality worldwide for 36 cancers in 185 countries. CA: a cancer journal for clinicians 2018;68(6):394-424. doi: 10.3322/caac.21492 [published Online First: 2018/09/13]

2. Machlowska J, Maciejewski R, Sitarz R. The Pattern of Signatures in Gastric Cancer Prognosis. International journal of molecular sciences 2018;19(6) doi: 10.3390/ijms19061658 [published Online First: 2018/06/06]

3. Ferro A, Peleteiro B, Malvezzi M, et al. Worldwide trends in gastric cancer mortality (1980-2011), with predictions to 2015, and incidence by subtype. European journal of cancer (Oxford, England : 1990) 2014;50(7):1330-44. doi: 10.1016/j.ejca.2014.01.029 [published Online First: 2014/03/22]

4. Siegel R, Ma J, Zou Z, et al. Cancer statistics, 2014. CA: a cancer journal for clinicians 2014;64(1):929. doi: 10.3322/caac. 21208 [published Online First: 2014/01/09]

5. Niccolai E, Taddei A, Prisco D, et al. Gastric cancer and the epoch of immunotherapy approaches. World journal of gastroenterology 2015;21(19):5778-93. doi: 10.3748/wjg.v21.i19.5778 [published Online First: 2015/05/29]

6. Orditura M, Galizia G, Sforza V, et al. Treatment of gastric cancer. World journal of gastroenterology 2014;20(7):1635-49. doi: 10.3748/wjg.v20.i7.1635 [published Online First: 2014/03/04]

7. Wang XZ, Zeng ZY, Ye X, et al. Interpretation of the development of neoadjuvant therapy for gastric cancer based on the vicissitudes of the NCCN guidelines. World journal of gastrointestinal oncology 2020;12(1):37-53. doi: 10.4251/wjgo.v12.i1.37 [published Online First: 2020/01/23]

8. Ji Q, Luo YQ, Wang WH, et al. Research advances in traditional Chinese medicine syndromes in cancer patients. Journal of integrative medicine 2016;14(1):12-21. doi: 10.1016/s20954964(16)60237-6 [published Online First: 2016/01/19]

9. Wang Z, Qi F, Cui Y, et al. An update on Chinese herbal medicines as adjuvant treatment of anticancer therapeutics. Bioscience trends 2018;12(3):220-39. doi: 10.5582/bst.2018.01144 [published Online First: 2018/07/18]

10. Li X, Yang G, Li X, et al. Traditional Chinese medicine in cancer care: a review of controlled clinical studies published in chinese. PloS one 2013;8(4):e60338. doi: 10.1371/journal.pone.0060338 [published Online First: 2013/04/06] 
11. Qi F, Zhao L, Zhou A, et al. The advantages of using traditional Chinese medicine as an adjunctive therapy in the whole course of cancer treatment instead of only terminal stage of cancer. Bioscience trends 2015;9(1):16-34. doi: 10.5582/bst.2015.01019 [published Online First: 2015/03/20]

12. Zhang J, Zhan Z, Wu J, et al. Relationship between EGF, TGFA, and EGFR Gene Polymorphisms and Traditional Chinese Medicine ZHENG in Gastric Cancer. Evidence-based complementary and alternative medicine : eCAM 2013;2013:731071. doi: 10.1155/2013/731071 [published Online First: 2014/01/24]

13. Hung KF, Hsu CP, Chiang JH, et al. Complementary Chinese herbal medicine therapy improves survival of patients with gastric cancer in Taiwan: A nationwide retrospective matched-cohort study. Journal of ethnopharmacology 2017;199:168-74. doi: 10.1016/j.jep.2017.02.004 [published Online First: 2017/02/07]

14. Song W, Ni S, Fu Y, et al. Uncovering the mechanism of Maxing Ganshi Decoction on asthma from a systematic perspective: A network pharmacology study. Scientific reports 2018;8(1):17362. doi: 10.1038/s41598-018-35791-9 [published Online First: 2018/11/28]

15. Huang Z, Wei P. Compound Kushen Injection for gastric cancer: A protocol of systematic review and meta-analysis. Medicine 2019;98(45):e17927. doi: 10.1097/md.0000000000017927 [published Online First: 2019/11/09]

16. Luo TT, Lu Y, Yan SK, et al. Network Pharmacology in Research of Chinese Medicine Formula: Methodology, Application and Prospective. Chinese journal of integrative medicine 2020;26(1):72-80. doi: 10.1007/s11655-019-3064-0 [published Online First: 2019/04/04]

17. Zhang W, Huai Y, Miao Z, et al. Systems Pharmacology for Investigation of the Mechanisms of Action of Traditional Chinese Medicine in Drug Discovery. Frontiers in pharmacology 2019;10:743. doi: 10.3389/fphar.2019.00743 [published Online First: 2019/08/06]

18. Ru J, Li P, Wang J, et al. TCMSP: a database of systems pharmacology for drug discovery from herbal medicines. Journal of cheminformatics 2014;6:13. doi: 10.1186/1758-2946-6-13 [published Online First: 2014/04/17]

19. Kim MT, Sedykh A, Chakravarti SK, et al. Critical evaluation of human oral bioavailability for pharmaceutical drugs by using various cheminformatics approaches. Pharmaceutical research 2014;31(4):1002-14. doi: 10.1007/s11095-013-1222-1 [published Online First: 2013/12/07]

20. Shen M, Tian S, Li Y, et al. Drug-likeness analysis of traditional Chinese medicines: 1. property distributions of drug-like compounds, non-drug-like compounds and natural compounds from traditional Chinese medicines. Journal of cheminformatics 2012;4(1):31. doi: 10.1186/1758-2946-431 [published Online First: 2012/11/28]

21. Safran M, Dalah I, Alexander J, et al. GeneCards Version 3: the human gene integrator. Database : the journal of biological databases and curation 2010;2010:baq020. doi: 10.1093/database/baq020 [published Online First: 2010/08/07]

22. Otasek D, Morris JH, Bouças J, et al. Cytoscape Automation: empowering workflow-based network analysis. Genome biology 2019;20(1):185. doi: 10.1186/s13059-019-1758-4 [published Online First: 
2019/09/04]

23. Shannon P, Markiel A, Ozier O, et al. Cytoscape: a software environment for integrated models of biomolecular interaction networks. Genome research 2003;13(11):2498-504. doi:

10.1101/gr.1239303 [published Online First: 2003/11/05]

24. Gene Ontology Consortium: going forward. Nucleic acids research 2015;43(Database issue):D104956. doi: 10.1093/nar/gku1179 [published Online First: 2014/11/28]

25. Kanehisa M, Goto S. KEGG: kyoto encyclopedia of genes and genomes. Nucleic acids research 2000;28(1):27-30. doi: 10.1093/nar/28.1.27 [published Online First: 1999/12/11]

26. Yang S, Zhang J, Yan Y, et al. Network Pharmacology-Based Strategy to Investigate the Pharmacologic Mechanisms of Atractylodes macrocephala Koidz. for the Treatment of Chronic Gastritis. Frontiers in pharmacology 2019;10:1629. doi: 10.3389/fphar.2019.01629 [published Online First: 2020/02/18]

27. Zhang J, Wu C, Gao L, et al. Astragaloside IV derived from Astragalus membranaceus: A research review on the pharmacological effects. Advances in pharmacology (San Diego, Calif) 2020;87:89112. doi: 10.1016/bs.apha.2019.08.002 [published Online First: 2020/02/25]

28. Hariri M, Ghiasvand R. Cinnamon and Chronic Diseases. Advances in experimental medicine and biology 2016;929:1-24. doi: 10.1007/978-3-319-41342-6_1 [published Online First: 2016/10/25]

29. Yang Y, Li SS, Teixeira da Silva JA, et al. Characterization of phytochemicals in the roots of wild herbaceous peonies from China and screening for medicinal resources. Phytochemistry 2020;174:112331. doi: 10.1016/j.phytochem.2020.112331 [published Online First: 2020/03/09]

30. Guo YJ, Pan WW, Liu SB, et al. ERK/MAPK signalling pathway and tumorigenesis. Experimental and therapeutic medicine 2020;19(3):1997-2007. doi: 10.3892/etm.2020.8454 [published Online First: 2020/02/28]

31. Olivier M, Hollstein M, Hainaut P. TP53 mutations in human cancers: origins, consequences, and clinical use. Cold Spring Harbor perspectives in biology 2010;2(1):a001008. doi:

10.1101/cshperspect.a001008 [published Online First: 2010/02/26]

32. Baker KJ, Houston A, Brint E. IL-1 Family Members in Cancer; Two Sides to Every Story. Frontiers in immunology 2019;10:1197. doi: 10.3389/fimmu.2019.01197 [published Online First: 2019/06/25]

33. Zhang W, Duan N, Song T, et al. The Emerging Roles of Forkhead Box (FOX) Proteins in Osteosarcoma. Journal of Cancer 2017;8(9):1619-28. doi: 10.7150/jca.18778 [published Online First: 2017/08/05]

34. Cully M, You H, Levine AJ, et al. Beyond PTEN mutations: the PI3K pathway as an integrator of multiple inputs during tumorigenesis. Nature reviews Cancer 2006;6(3):184-92. doi: 10.1038/nrc1819 [published Online First: 2006/02/03]

35. Chang F, Lee JT, Navolanic PM, et al. Involvement of PI3K/Akt pathway in cell cycle progression, apoptosis, and neoplastic transformation: a target for cancer chemotherapy. Leukemia 2003;17(3):590-603. doi: 10.1038/sj.leu.2402824 [published Online First: 2003/03/21] 
36. Chen ZF, Wang J, Yu Y, et al. MicroRNA-936 promotes proliferation and invasion of gastric cancer cells by down-regulating FGF2 expression and activating P13K/Akt signaling pathway. European review for medical and pharmacological sciences 2020;24(12):6707-15. doi:

10.26355/eurrev_202006_21658 [published Online First: 2020/07/08]

37. Riquelme I, Tapia O, Espinoza JA, et al. The Gene Expression Status of the PI3K/AKT/mTOR Pathway in Gastric Cancer Tissues and Cell Lines. Pathology oncology research : POR 2016;22(4):797-805. doi: 10.1007/s12253-016-0066-5 [published Online First: 2016/05/09]

38. Oki E, Baba H, Tokunaga E, et al. Akt phosphorylation associates with LOH of PTEN and leads to chemoresistance for gastric cancer. International journal of cancer 2005;117(3):376-80. doi: 10.1002/ijc.21170 [published Online First: 2005/05/19]

39. Yang SH, Sharrocks AD, Whitmarsh AJ. MAP kinase signalling cascades and transcriptional regulation. Gene 2013;513(1):1-13. doi: 10.1016/j.gene.2012.10.033 [published Online First: 2012/11/06]

40. Wagner EF, Nebreda AR. Signal integration by JNK and p38 MAPK pathways in cancer development. Nature reviews Cancer 2009;9(8):537-49. doi: 10.1038/nrc2694 [published Online First: 2009/07/25]

41. Dhillon AS, Hagan S, Rath O, et al. MAP kinase signalling pathways in cancer. Oncogene 2007;26(22):3279-90. doi: 10.1038/sj.onc.1210421 [published Online First: 2007/05/15]

42. Davies $\mathrm{H}$, Bignell GR, Cox $\mathrm{C}$, et al. Mutations of the BRAF gene in human cancer. Nature 2002;417(6892):949-54. doi: 10.1038/nature00766 [published Online First: 2002/06/18]

43. Karabulut M, Usul Afsar C, Serimez M, et al. Serum IL-17 levels can be diagnostic for gastric cancer. Journal of BUON : official journal of the Balkan Union of Oncology 2019;24(4):1601-09. [published Online First: 2019/10/28]

44. Xu J, Lv S, Meng W, et al. LCN2 Mediated by IL-17 Affects the Proliferation, Migration, Invasion and Cell Cycle of Gastric Cancer Cells by Targeting SLPI. Cancer management and research 2020;12:12841-49. doi: 10.2147/cmar.S278902 [published Online First: 2020/12/29]

45. Bastid J, Dejou C, Docquier A, et al. The Emerging Role of the IL-17B/IL-17RB Pathway in Cancer. Frontiers in immunology 2020;11:718. doi: 10.3389/fimmu.2020.00718 [published Online First: 2020/05/07]

46. Huang WJ, Ruan S, Wen F, et al. Multidrug Resistance of Gastric Cancer: The Mechanisms and Chinese Medicine Reversal Agents. Cancer management and research 2020;12:12385-94. doi: 10.2147/cmar.S274599 [published Online First: 2020/12/10]

47. Yang L, Hu Z, Zhu J, et al. Systematic Elucidation of the Mechanism of Quercetin against Gastric Cancer via Network Pharmacology Approach. BioMed research international 2020;2020:3860213. doi: 10.1155/2020/3860213 [published Online First: 2020/09/24]

48. Wang K, Liu R, Li J, et al. Quercetin induces protective autophagy in gastric cancer cells: involvement of Akt-mTOR- and hypoxia-induced factor 1a-mediated signaling. Autophagy 2011;7(9):966-78. doi: 10.4161/auto.7.9.15863 [published Online First: 2011/05/26] 
49. Li H, Chen C. Quercetin Has Antimetastatic Effects on Gastric Cancer Cells via the Interruption of UPA/uPAR Function by Modulating NF-Kb, PKC-ס, ERK1/2, and AMPKa. Integrative cancer therapies 2018;17(2):511-23. doi: 10.1177/1534735417696702 [published Online First: 2017/06/20]

50. Granado-Serrano AB, Martín MA, Bravo L, et al. Quercetin induces apoptosis via caspase activation, regulation of $\mathrm{Bcl}-2$, and inhibition of $\mathrm{PI}-3$-kinase/Akt and ERK pathways in a human hepatoma cell line (HepG2). The Journal of nutrition 2006;136(11):2715-21. doi: 10.1093/jn/136.11.2715 [published Online First: 2006/10/24]

51. Niu G, Yin S, Xie S, et al. Quercetin induces apoptosis by activating caspase-3 and regulating Bcl-2 and cyclooxygenase-2 pathways in human HL-60 cells. Acta biochimica et biophysica Sinica 2011;43(1):30-7. doi: 10.1093/abbs/gmq107 [published Online First: 2010/12/22]

52. Lee DH, Szczepanski M, Lee YJ. Role of Bax in quercetin-induced apoptosis in human prostate cancer cells. Biochemical pharmacology 2008;75(12):2345-55. doi: 10.1016/j.bcp.2008.03.013 [published Online First: 2008/05/06]

53. Borska S, Chmielewska M, Wysocka T, et al. In vitro effect of quercetin on human gastric carcinoma: targeting cancer cells death and MDR. Food and chemical toxicology : an international journal published for the British Industrial Biological Research Association 2012;50(9):3375-83. doi: 10.1016/j.fct.2012.06.035 [published Online First: 2012/07/04]

54. Shang HS, Lu HF, Lee CH, et al. Quercetin induced cell apoptosis and altered gene expression in AGS human gastric cancer cells. Environmental toxicology 2018;33(11):1168-81. doi: 10.1002/tox.22623 [published Online First: 2018/08/29]

55. Chen AY, Chen YC. A review of the dietary flavonoid, kaempferol on human health and cancer chemoprevention. Food chemistry 2013;138(4):2099-107. doi: 10.1016/j.foodchem.2012.11.139 [published Online First: 2013/03/19]

56. Luo H, Daddysman MK, Rankin GO, et al. Kaempferol enhances cisplatin's effect on ovarian cancer cells through promoting apoptosis caused by down regulation of cMyc. Cancer cell international 2010;10:16. doi: 10.1186/1475-2867-10-16 [published Online First: 2010/05/13]

57. Chen HJ, Lin CM, Lee CY, et al. Kaempferol suppresses cell metastasis via inhibition of the ERK-p38JNK and AP-1 signaling pathways in U-2 OS human osteosarcoma cells. Oncology reports 2013;30(2):925-32. doi: 10.3892/or.2013.2490 [published Online First: 2013/05/28]

58. Choi EJ, Ahn WS. Kaempferol induced the apoptosis via cell cycle arrest in human breast cancer MDA-MB-453 cells. Nutrition research and practice 2008;2(4):322-5. doi: 10.4162/nrp.2008.2.4.322 [published Online First: 2008/01/01]

59. Song W, Dang Q, Xu D, et al. Kaempferol induces cell cycle arrest and apoptosis in renal cell carcinoma through EGFR/p38 signaling. Oncology reports 2014;31(3):1350-6. doi: 10.3892/or.2014.2965 [published Online First: 2014/01/09]

60. Yang L, Li H, Yang M, et al. Exploration in the Mechanism of Kaempferol for the Treatment of Gastric Cancer Based on Network Pharmacology. BioMed research international 2020;2020:5891016. doi: 10.1155/2020/5891016 [published Online First: 2020/11/05] 
61. Song $\mathrm{H}, \mathrm{Bao} J$, Wei $\mathrm{Y}$, et al. Kaempferol inhibits gastric cancer tumor growth: An in vitro and in vivo study. Oncology reports 2015;33(2):868-74. doi: 10.3892/or.2014.3662 [published Online First: 2014/12/17]

62. Gong G, Guan YY, Zhang ZL, et al. Isorhamnetin: A review of pharmacological effects. Biomedicine \& pharmacotherapy $=$ Biomedecine \& pharmacotherapie 2020;128:110301. doi:

10.1016/j.biopha.2020.110301 [published Online First: 2020/06/06]

63. Ramachandran L, Manu KA, Shanmugam MK, et al. Isorhamnetin inhibits proliferation and invasion and induces apoptosis through the modulation of peroxisome proliferator-activated receptor $Y$ activation pathway in gastric cancer. The Journal of biological chemistry 2012;287(45):38028-40. doi: 10.1074/jbc.M112.388702 [published Online First: 2012/09/21]

64. Pu Y, Zhang T, Wang J, et al. Luteolin exerts an anticancer effect on gastric cancer cells through multiple signaling pathways and regulating miRNAs. Journal of Cancer 2018;9(20):3669-75. doi: 10.7150/jca.27183 [published Online First: 2018/11/09]

65. Zang MD, Hu L, Fan ZY, et al. Luteolin suppresses gastric cancer progression by reversing epithelialmesenchymal transition via suppression of the Notch signaling pathway. Journal of translational medicine 2017;15(1):52. doi: 10.1186/s12967-017-1151-6 [published Online First: 2017/03/01]

\section{Figures}




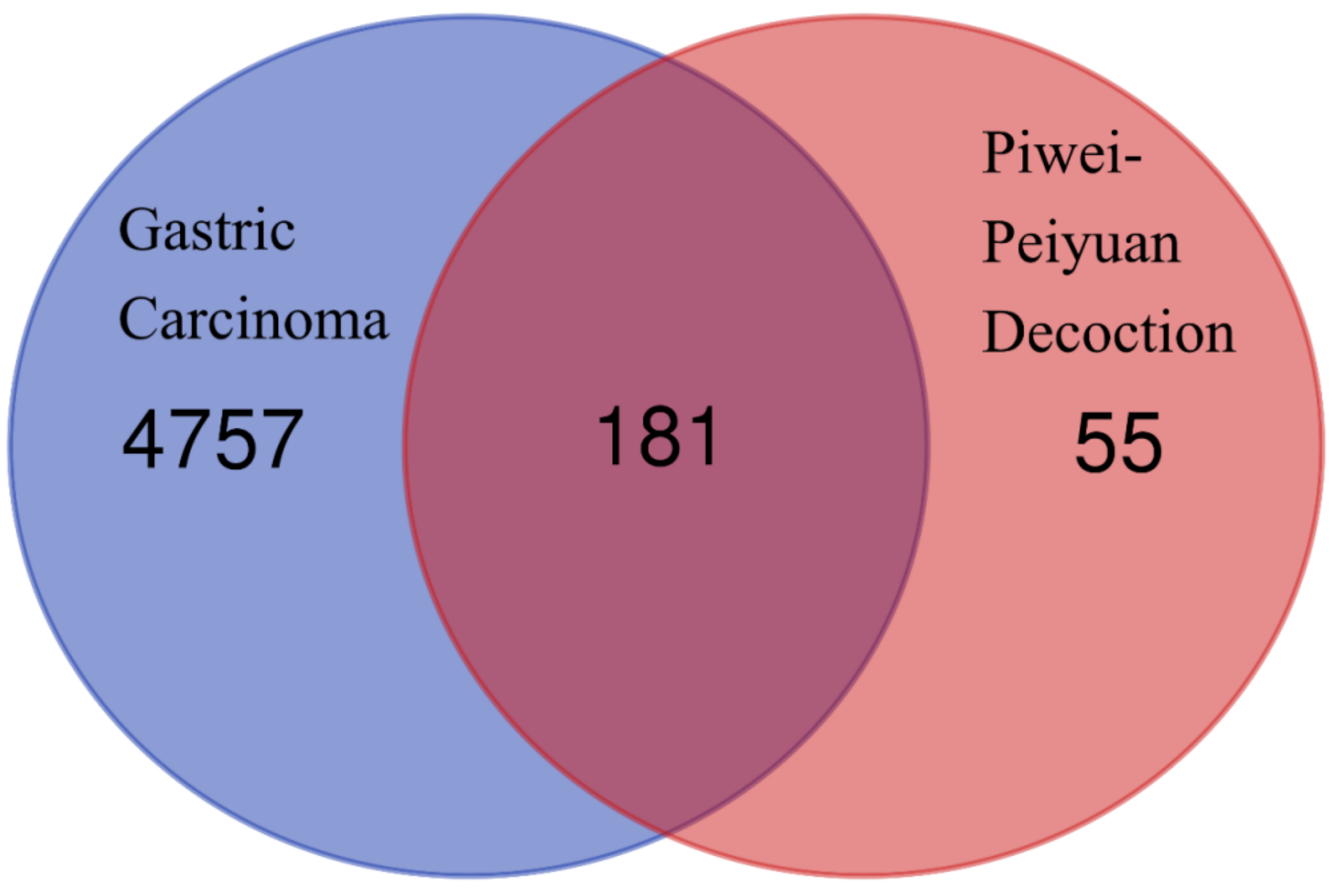

Figure 1

Venn diagram. Note: We found 4938 tagets genes of GC and 236 targets of Piwei-Peiyuan Decoction. 181 common targets are common targets. 


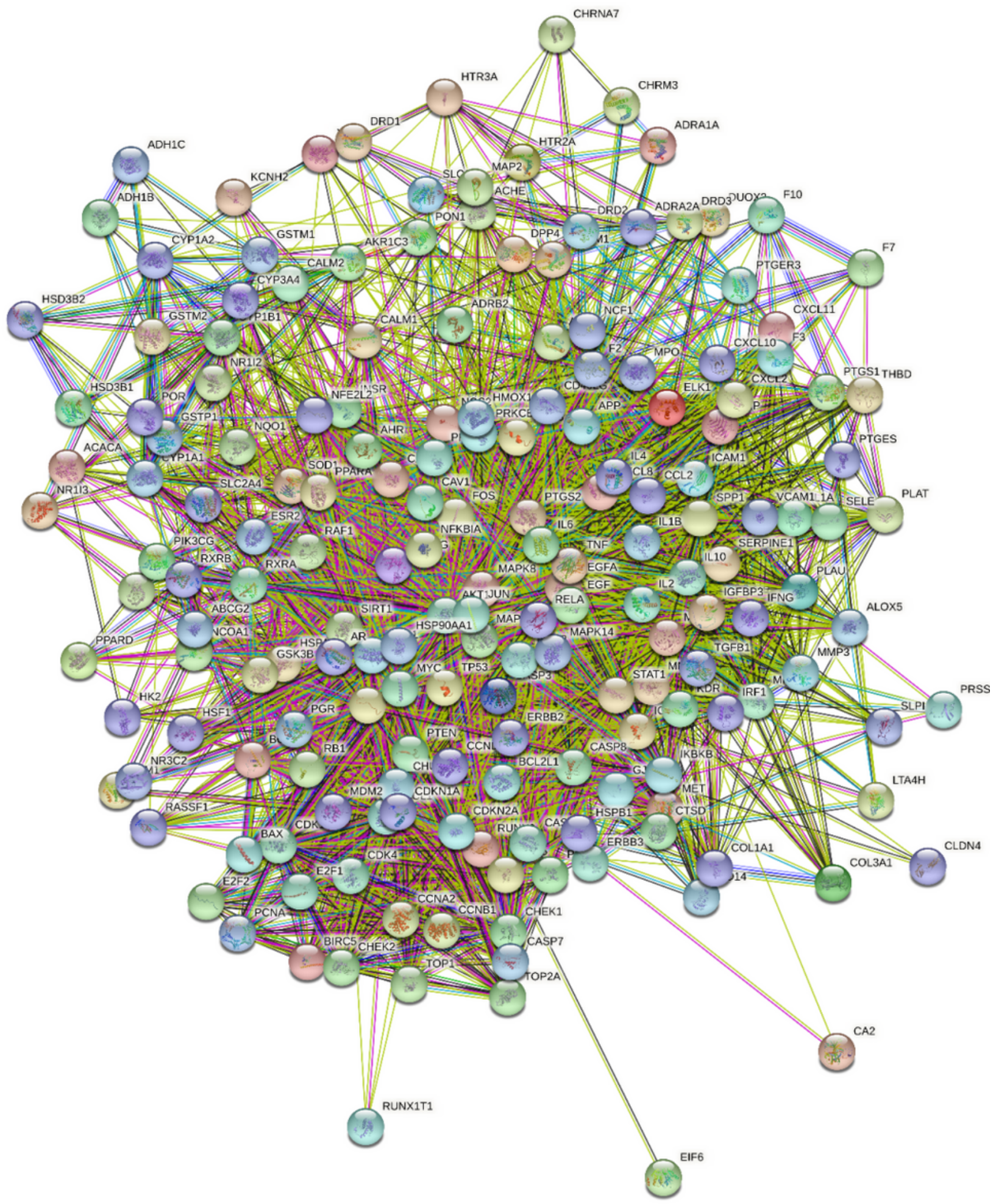

Figure 2

Protein-protein Interaction (PPI) Network. 


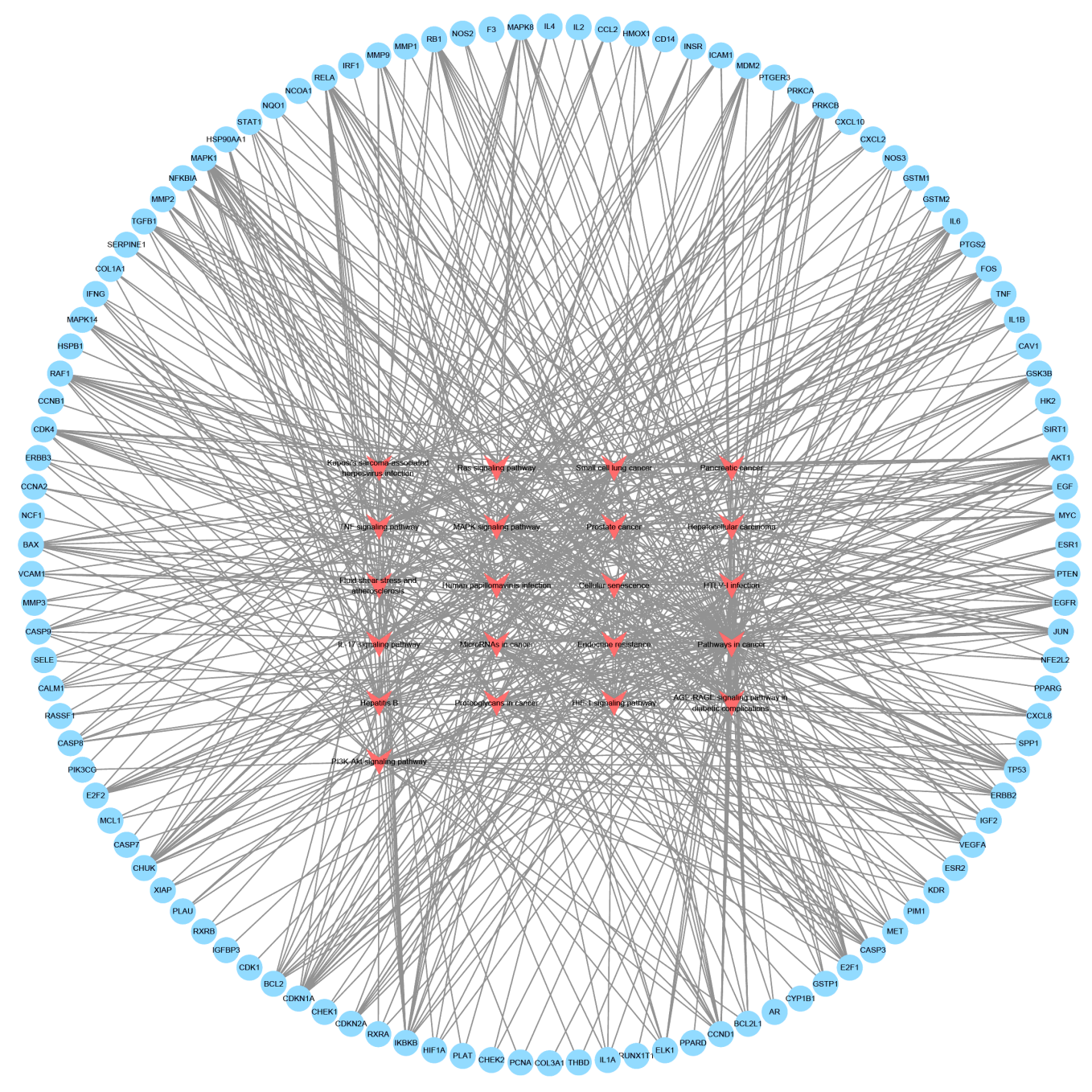

Figure 3

Targets-Pathways Interaction Network. 


\section{Gene Ontology Analysis}

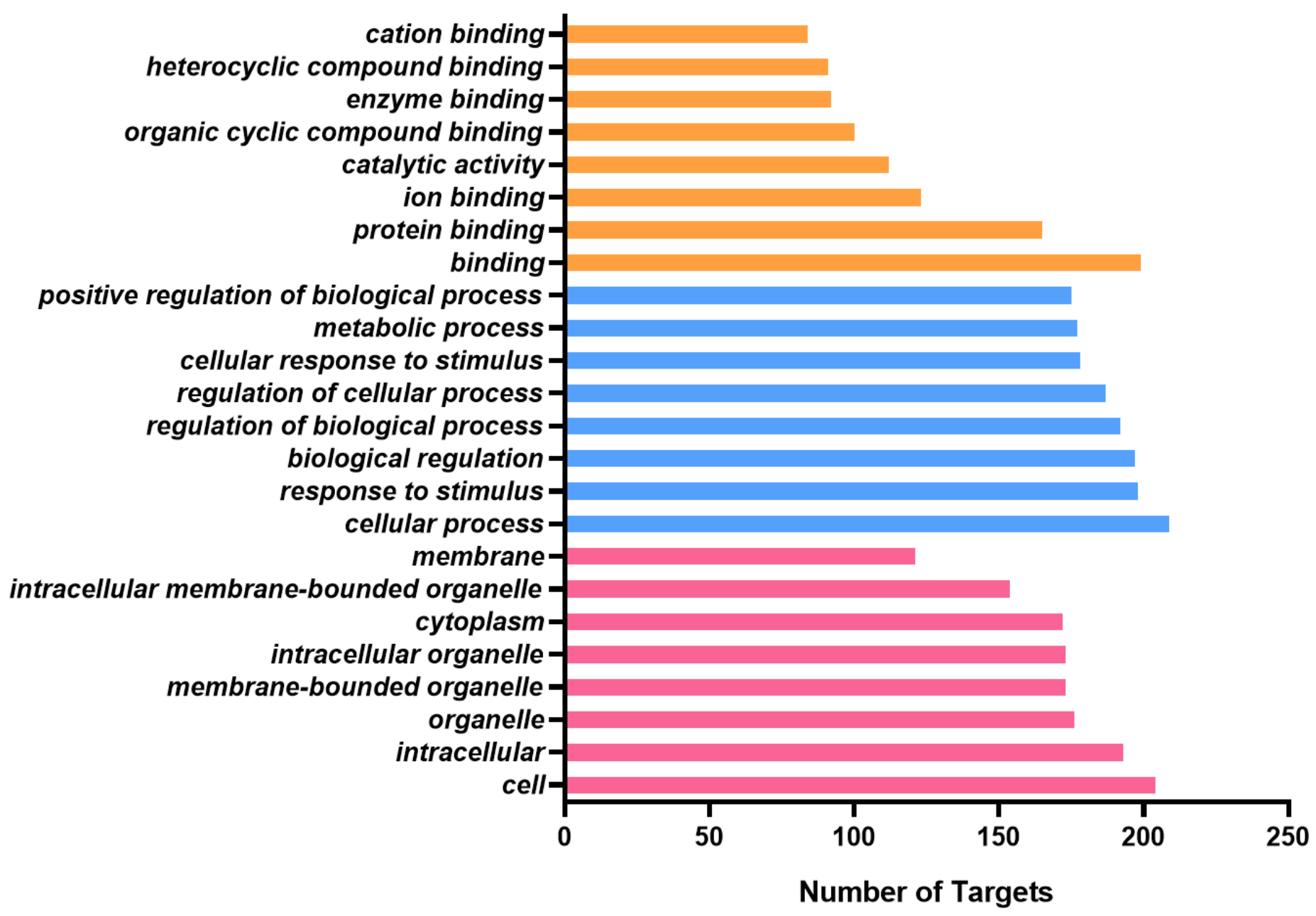

Figure 4

Gene Ontology Analysis of Targets Genes 


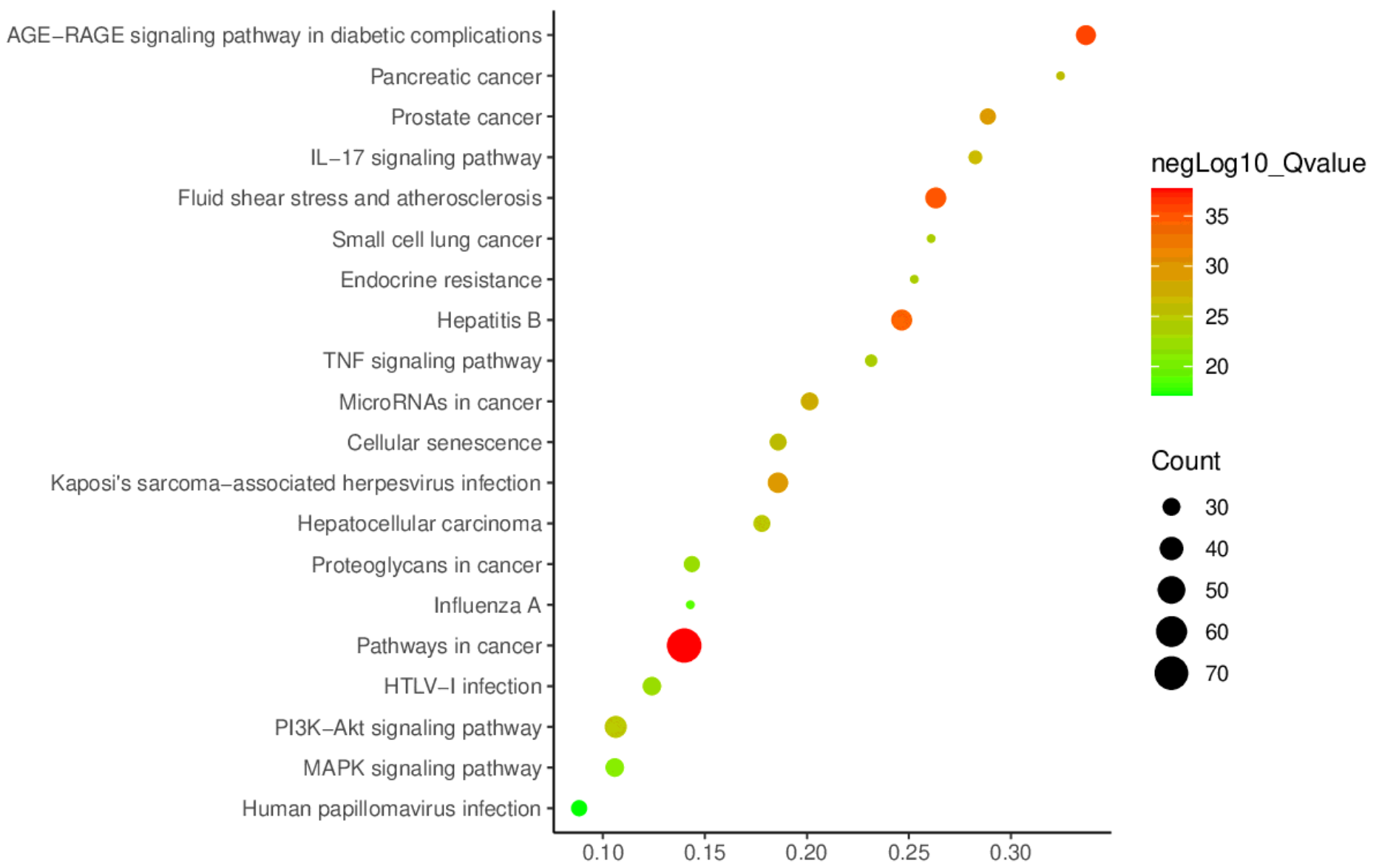

Figure 5

KEGG Pathway Analysis of Target Genes 


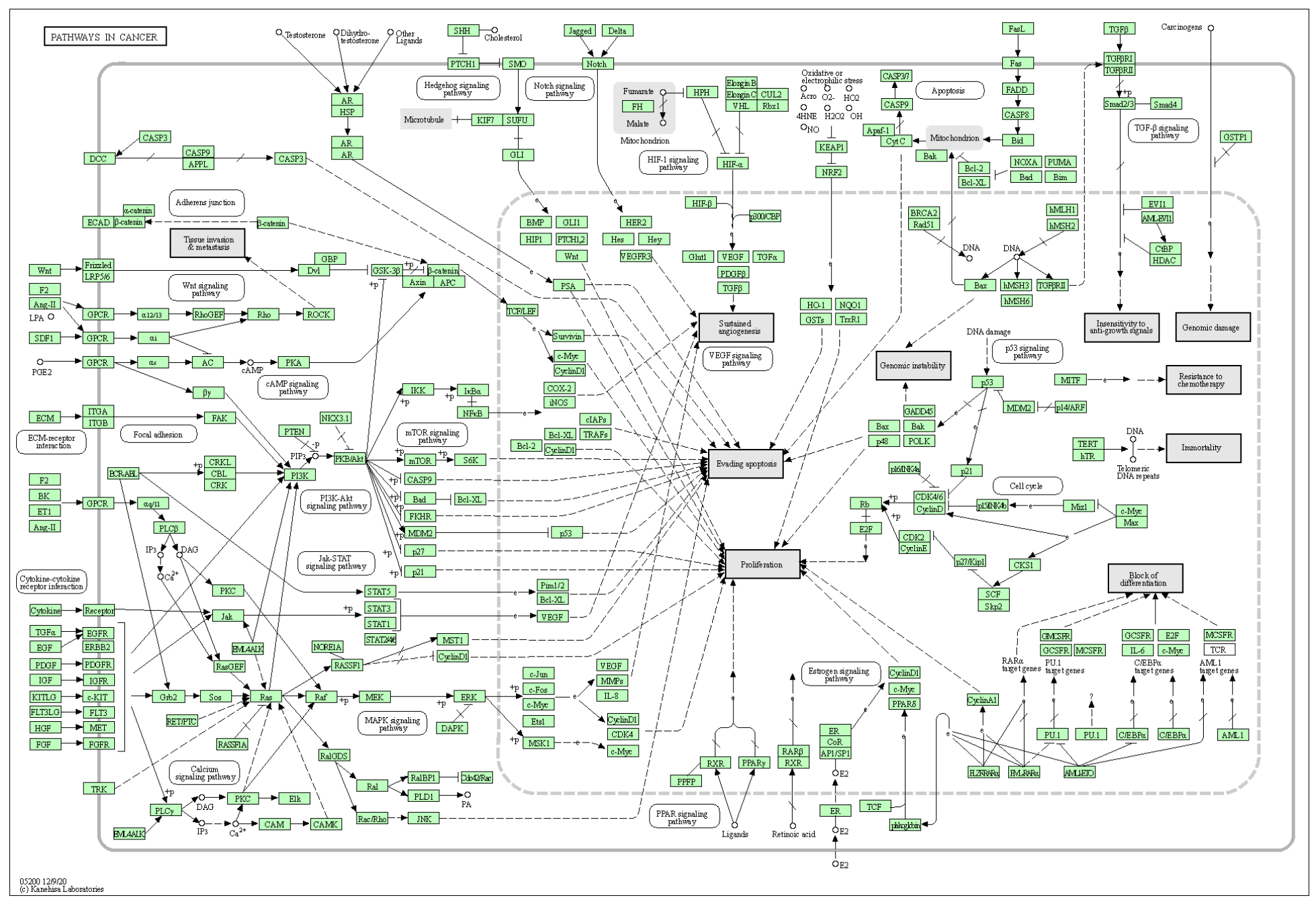

Figure 6

Network of Pathway in Cancer Signal Pathway 


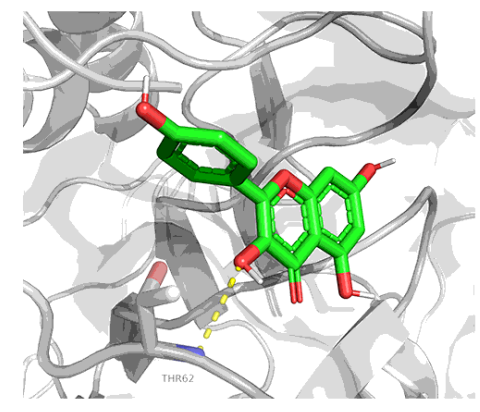

CASP3-Kaempferol

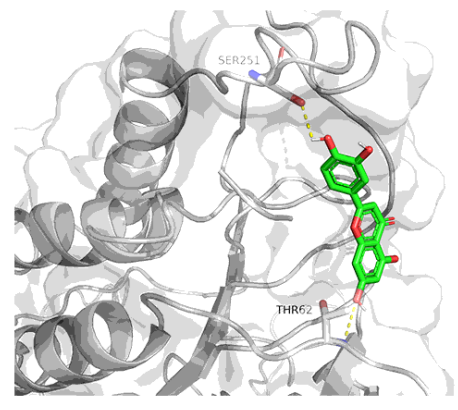

CASP3-Luteolin

(a)

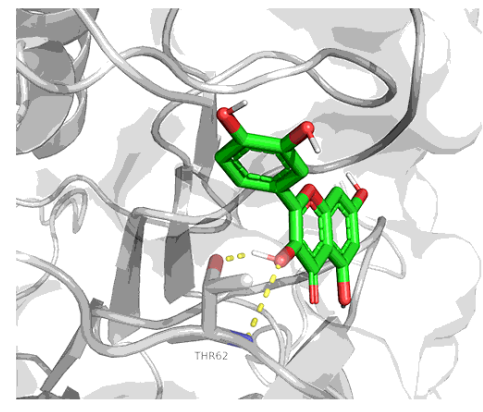

CASP3-Quercetin

(c)

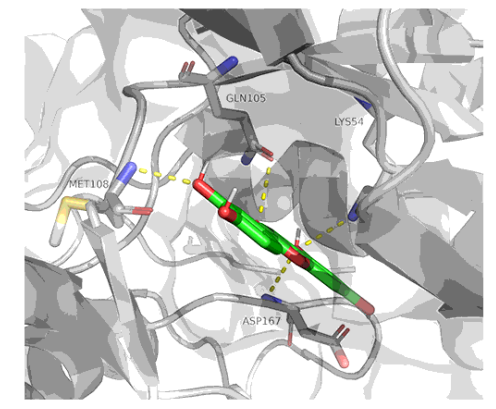

MAPK1-Luteolin

(d)

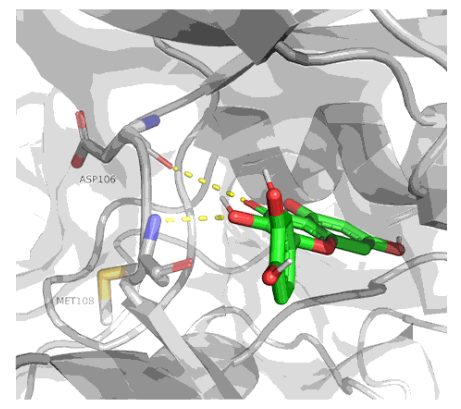

MAPK1-Quercetin

(e)

\section{Figure 7}

Molecular Docking of Key Targets and Ingredients. Note: Docking concept and binding site analysis of Kaempfelol (a). Docking concept and binding site analysis of Luteolin (b, d). Docking concept and binding site analysis of Quercetin (c,e). 